\title{
Dynamics of Landing a Spacecraft on a Celestial Body with Small Field of Gravity
}

\author{
Victor P. Kazakovtsev ${ }^{a}$, Vsevolod V. Koryanov ${ }^{c}$, and Chen Danhe ${ }^{b}$ \\ 2-nd Baumanskaya str., 5, Build 1, Moscow, Russian Federation, Bauman Moscow State \\ Technical University \\ akafsm3@bmstu.ru, bvoryanov@mail.ru, ccranefeeling@hotmail.com
}

Keywords: Spacecraft, dynamics of landing, modeling, landing vehicle, dynamics, the descent into the atmosphere.

Abstract. Currently most of the researches on the movement of space lander is based on landing on celestial bodies with a significant field of gravity, this paper considers the problem of the dynamics of landing on the surface of a celestial body with a small field of gravity.

\section{Introduction}

The study of problems of landing on the other celestial bodies in the solar system started fifty years ago. A sufficient amount of work was devoted to the problems of landing on the moon, for example, in $[1,2]$ have made an analysis of the stability of motion of space lander when landing on the moon. Now we are considering about the problems of landing on small celestial bodies (asteroids, comets, small satellites of planets, etc.), and one problem was proposed, which is about the landing and fixation space lander to the surface of such bodies. In the last space mission the lander Rosetta departed from the surface of comet several times. In this paper presents a sustainable solution to the problems of landing on a small celestial body using a special penetrator anchor.

\section{Discussed Problems}

The movement of space lander is considered in the surface coordinate system (UCS). The beginning of UCS is located on the surface of the celestial body. Axis OX goes through the left leg of space lander and locates in the horizontal plane. Axis OY is perpendicular to horizontal plane. Axis OZ is defined by right-handed system. In accordance with the work [3], the dynamic equations of motion by the center mass of the space lander and the dynamic equations of rotation are shown as follows:

$$
\begin{aligned}
& m \frac{d V_{x}}{d t}=\sum_{j=1}^{n} F_{T x j}+T_{x}+P_{\partial \delta x}+m g_{x} \\
& m \frac{d V_{y}}{d t}=\sum_{j=1}^{n} F_{N j}+T_{y}+P_{\partial \delta y}+m g_{y} \\
& m \frac{d V_{z}}{d t}=\sum_{j=1}^{n} F_{T z j}+T_{z}+P_{\partial \delta z}+m g_{z} \\
& J_{y} \frac{d \omega_{y}}{d t}=\sum_{j=1}^{n} M_{y c j}+M_{T c y}+\left(J_{z}-J_{x}\right) \omega_{x} \omega_{z} \\
& J_{z} \frac{d \omega_{z}}{d t}=\sum_{j=1}^{n} M_{z c j}+M_{T c z}+\left(J_{x}-J_{y}\right) \omega_{y} \omega_{x} \\
& J_{x} \frac{d \omega_{x}}{d t}=\sum_{j=1}^{n} M_{x c j}+M_{T c x}+\left(J_{y}-J_{z}\right) \omega_{y} \omega_{z}
\end{aligned}
$$


where: $F_{T x j}, F_{T y j}, F_{T z j}$-projection forces of reaction on the ground support,

$T x, T y, T z$ - projection forces of the cable tension (Tp);

Рдвх , Рдву,$Р \partial в z$ - projection forces of pinch engines;

$m g x, m g y, m g z,-$ projection forces of attraction;

$J x, J y, J z$-principal moments of inertia;

$\omega x, \omega y, \omega z$-the projection of the angular velority of the rotation of space lander on axises in related coordinate system;

$M_{T c x}, M_{T c y}, M_{T c z}$-projection of moments of cable tension on the axis in related coordinate system;

$M_{x c j}, M_{y c j}, M_{z c j}$ projection of moments of the forces acting on the $\mathrm{j}$-th support on axis in related coordinate system,

We suppose that all the supports have the same absorber step type with maximum force Fo. Regarding to the obtained value of deformation, in accordance with the schedule change in absorber force, we could define the value of the force $(F L j)$ :

If $\delta j<\delta j k$, то $F L j=0$, (before the first touch, we suppose that $\delta j k=0$ ),

If $\delta j>\delta j k$ и $\delta j<\delta j k 1$, then $F L j=F o(\delta j-\delta j k 1) / \delta$,

If $\delta j>\delta j k 1$, then $F L j=F o$,

where: $\delta$ o -the value of elastic deformation;

$\delta j k 1$-maximum deformation of absorber when a given support touch surface;

$\delta j k$-value of deformation of support after the departure from surface.

\section{Computational Examples and Analysis}

Considering the example of landing on the surface of the Martian satellite Phobos. The main design parameters of the space lander with three supports are shown as following:

$m=900 \mathrm{~kg}, J_{y}=300 \mathrm{kgm}^{2}, J_{x}=900 \mathrm{kgm}^{2}, J_{z}=900 \mathrm{kgm}^{2}, \delta_{o}=0,001 \mathrm{~m}, F_{0}=4000 \mathrm{~N}$.

The initial conditions of motion of space lander: $X=Z=0, Y=1.5 \mathrm{~m}, V_{y}=-1 \mathrm{~m} / \mathrm{s}, V_{x}=0,1 \mathrm{~m} / \mathrm{s}, V_{z}=0$, $\theta=-4^{0}, \psi=\gamma=0, \omega_{x}=\omega_{y}=\omega_{z}=0$.

Calculations by equations (1) for lack of cable tension $(T=0)$ are presented in figure 1 and figure 2.

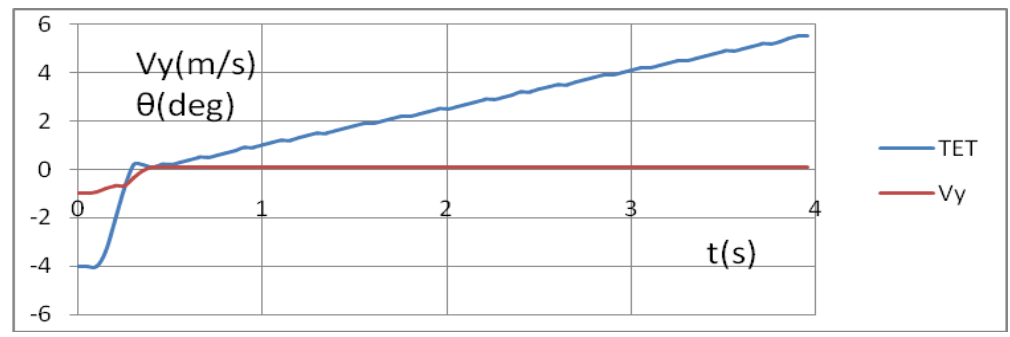

Fig. 1. Graphics of speed (Vy) and angle $(\theta)$ of space lander in the absence of cable tension.

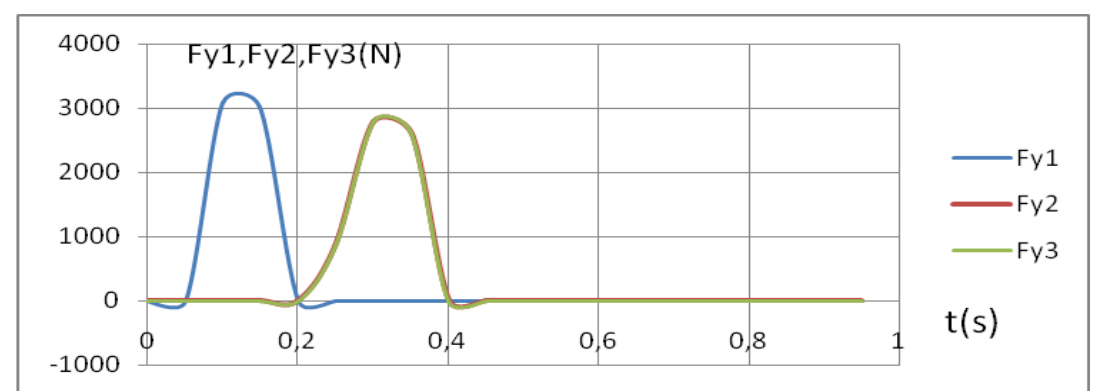

Fig. 2. Graphics of the vertical forces on supports in process of landing.

The figures show clearly that in the presence of angle $\theta$, space lander firstly touches the surface of Phobos by the first support, then both the second and third supports at one moment touch down. And then space lander departs away from the surface with increasing value of the angle $\theta$. 
Therefore, there will be not fix of space lander on the surface of Phobos. If we consider the case that the first support touches the surface of Phobos pulling by a cable, and the value of pulling is $T=500$ N. These results of calculation are shown in Fig. 3 and Fig. 4.

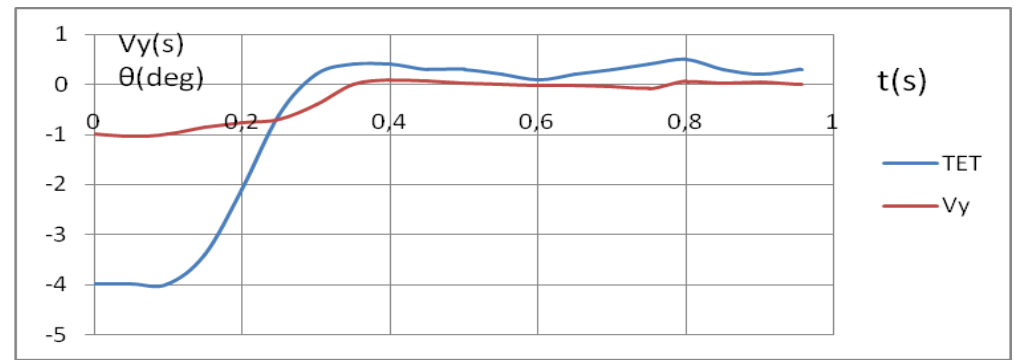

Fig. 3. Graphics of speed $(\mathrm{Vy})$ and angle $(\theta)$ of the space lander at $\mathrm{T}=500 \mathrm{~N}$

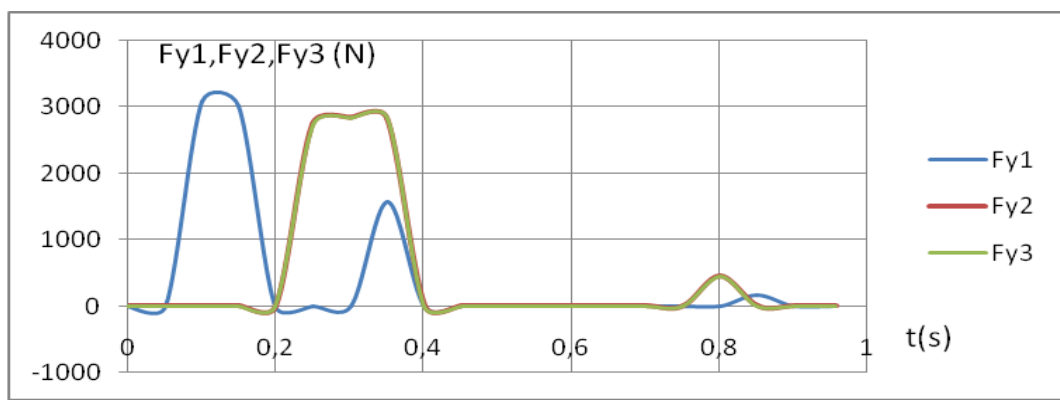

Fig. 4. Graphics of vertical forces on the supports of the space lander at $\mathrm{T}=500 \mathrm{~N}$

Analysis of results of the calculations by the equations (1) showed that in the presence of the angle $\theta=-4^{0}$ and velocity $V_{x}=0,1 \mathrm{~m} / \mathrm{s}$, the first support of space lander firstly goes close to the surface of Phobos, and the second and third support touch the surface. The cable tension make the first support to touch the surface again. As a result, the space lander turns around and the angle $\theta$ becomes practically zero, as well as the vertical velocity. Therefore, the presence of cable tesion for $500 \mathrm{~N}$ provides landing process of space lander without a rebound from the surface of a celestial body.

Consider another example of landing space lander on the surface of the Martian satellite Phobos. Technical Data Space lander with three legs are the same.

The initial conditions of orbital lander: $X=Z=0, Y=1.5 \mathrm{~m}, V_{y}=-1 \mathrm{~m} / \mathrm{s}, V_{x}=0, V_{z}=0, \psi=\gamma=0, \omega_{x}=\omega_{y}=$ $\omega_{z}=0$. Landing surface is inclined relative to the direction of movement of the space lander on the angle $\theta=5^{0}$. Calculations by equations (1) absence of options for cable tension $(T=0)$ are plotted in Fig. 5 and Fig 6.

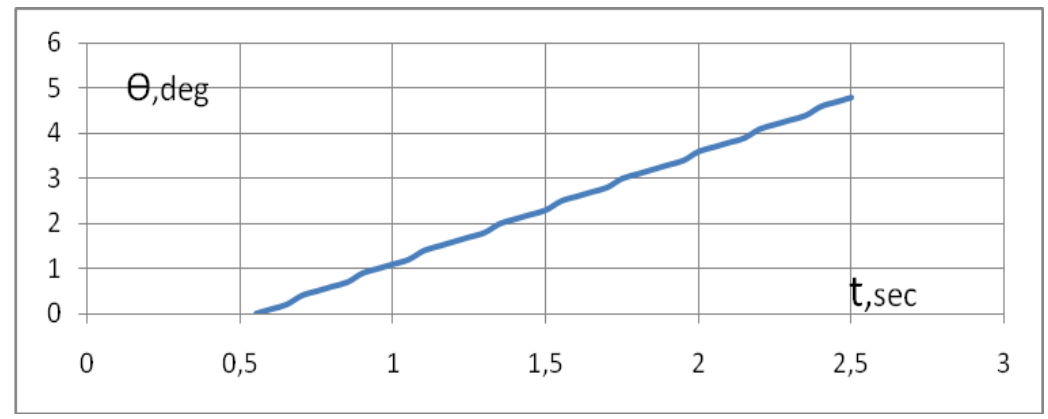

Fig. 5. Graphic angle of space lander in the absence of cable tension. 


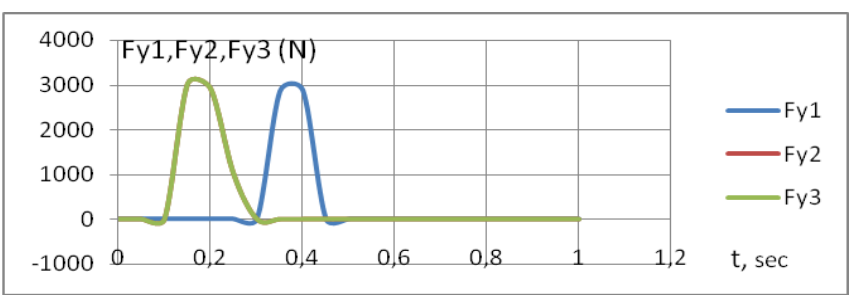

Fig. 6. Graphics of vertical forces on the pillars during landing space lander.

The figures show that in the presence of the surface angle to the direction of movement of the space lander touches the surface of Phobos first pillar, then both the second and third pillars. Then space lander moves away from the surface with the increasing value of the angle $\Theta$. Thus, the fixing of the space lander on the surface of Phobos will not.

Consider the case when at the touch of the first pillar of the surface of Phobos, the cable is pulled with a force $T=500 \mathrm{~N}$. These calculations taking into account the inclination of the surface to the direction of movement of the space lander are shown in Fig. 7 and Fig. 8.

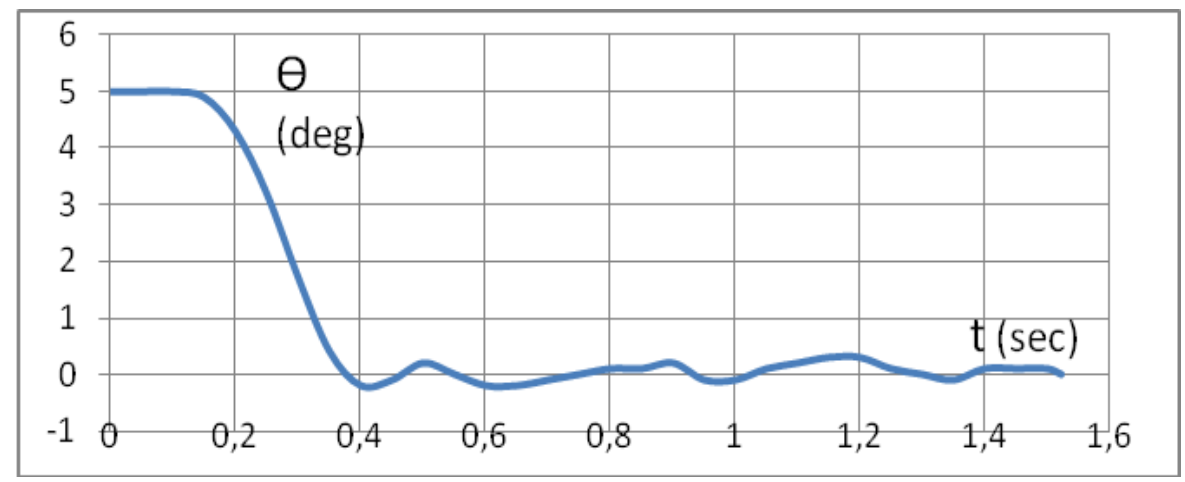

Fig. 7. Graphic of angle $(\theta)$ of space lander at $T=500 \mathrm{~N}$.

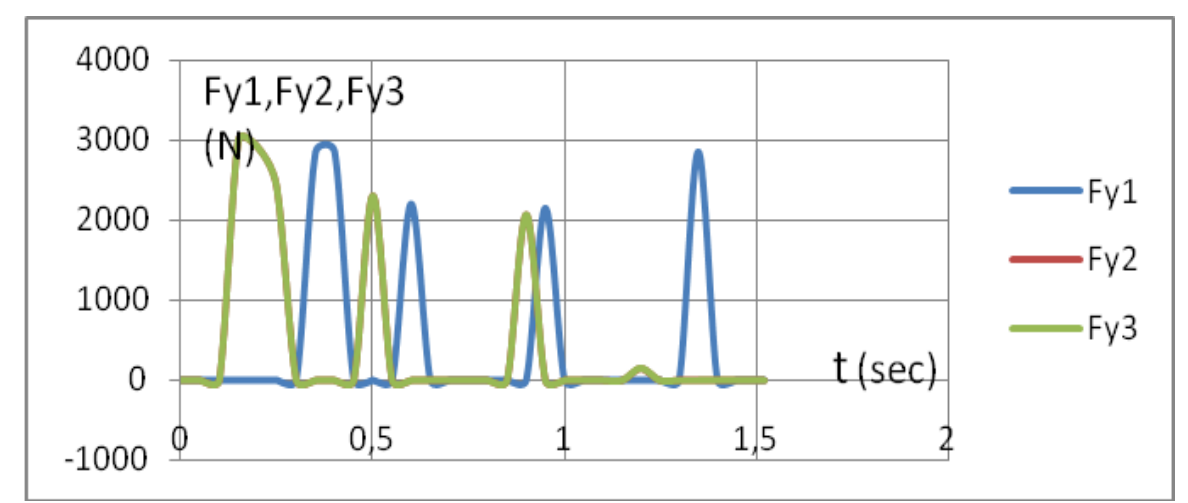

Fig. 8. Graphics of vertical forces on the pillars of the space lander landing on an inclined surface, and the wire tension $T=500 \mathrm{~N}$.

Analysis of the results of calculations showed that in the presence of a surface angle of 5 degree for space lander is close to the surface of Phobos, first second and third pillars on which the forces Fy 2 and Fy3. Then these supports depart, but the first pillar touches the surface. Cable tension forces again to touch the surface of the support. As a result, the space lander is set and the angle $\theta$ becomes practically zero.

Thus, the presence of cable tension force $500 \mathrm{~N}$ provides all space landers landing without bouncing on the inclined surface of a celestial body.

\section{Conclusions}


The proposed method using a special penetrator anchor with a cable landing on the surface of the small celestial body could significantly increase the reliability of landing. Analysis of the dynamics of motion of the space lander in the presence of a cable system showed that the process of landing has character of damping. The presence of various disturbing factors counters the initial tension force of cable.

\section{References}

[1] Kovtunenko V.M. Proektirovanie spuskaemykh avtomaticheskikh kosmicheskikh apparatov [Constructional design of landing unmanned space vehicles]. Moscow, Mashinostronie Publ., 1985. $264 \mathrm{p}$.

[2] Bazhenov V.I., Osin M.S. Posadka kosmicheskikh apparatov na planet [Spacecraft landing on the planets]. Moscow, Mashinostronie Publ., 1978, 158 p.

[3] Vicror P. Kazakovtsev, Chen Danhe, Vsevolod V. Koryanov. Issledovaniye dinamiki uglovogo dvizheniya kosmicheskogo posadochnogo apparata pri yego posadke na nebesnoye telo $\mathrm{s}$ malym polem tyagoteniya [Study of the dynamics of the angular motion of a space landing vehicle during its landing on a celestial body with a small field of gravity] //Obshcherossiyskiy nauchno-tekhnicheskiy zhurnal [Flight. All-Russ. Sc. - Techn. Journ.)], 2014, no.10, pp. 47 53 (in Russ.). 